\title{
Contender waveforms for Low-Power Wide-Area networks in a scheduled 4G OFDM framework
}

\author{
Yoann Roth ${ }^{1,2}$, Jean-Baptiste Doré ${ }^{*}$, Laurent $\operatorname{Ros}^{1}$ and Vincent Berg ${ }^{2}$
}

\begin{abstract}
When designing new solutions for Low-Power Wide-Area (LPWA) networks, coexistence and integration into existing 4G frameworks should be considered to ease the deployment procedure and reduce costs. In a previous work, Turbo-FSK was proposed as a potential physical layer for LPWA networks. With its constant envelope and high energy efficiency, the scheme is a serious contender for this type of networks. We propose to study the system in the Orthogonal Frequency Division Multiplexing (OFDM) framework, currently used by existing cellular networks and also considered for the recently standardized Narrow-Band IoT (NB-loT). Several extensions of the Turbo-FSK scheme are presented. A hybrid modulation alphabet, combining orthogonal with linear modulations, is introduced, and the substitution of Frequency Shift Keying (FSK) modulation for another orthogonal modulation based on Zadoff-Chu (ZC) sequences is considered. Simulations are run under various conditions including Rayleigh fading channel with mobility, demonstrating that the Turbo-FSK scheme is able to achieve performance close to the Turbo Coded Orthogonal Frequency Division Multiplexing (TC-OFDM) when a low rate is considered. When considering hybridation of the alphabet, limitations appear for higher data rate, which can be overcome by changing the orthogonal alphabet. The study of the variation of the envelope of the compared solutions emphasizes the crucial trade-off between performance and efficiency of the power amplifier (PA), a main concern for low-power applications. While using the proposed solutions, we demonstrate that energy consumption can be reduced by up to a factor of 2.5 .
\end{abstract}

Keywords: LPWA, OFDM, Turbo codes, FSK, Zadoff-Chu, Constant envelope

\section{Introduction}

The Internet of Things (IoT) is expected to interconnect objects using both existing communication technologies and new emerging technologies $[1,2]$. These new technologies should be designed in a way that facilitates integration to existing frameworks, e.g, the Orthogonal Frequency Division Multiplexing (OFDM) framework used in Long-Term Evolution (LTE) systems (4G). The framework defines the structure of the signal and the overall parameters of the physical waveforms. By considering an existing framework, deployment costs are reduced and coexistence between various technologies is facilitated.

Low-Power Wide-Area (LPWA) networks [3, 4] are part of the IoT and $5 \mathrm{G}$ context and are predicted to

*Correspondence: jean-baptiste.dore@cea.fr

${ }^{2}$ CEA, LETI, MINATEC Campus, 38054 Grenoble, France

Full list of author information is available at the end of the article approximately represent $10 \%$ of the overall IoT connections [5]. Requirements for LPWA include longrange communication and low-energy consumption at the device level. Long range can be achieved by ensuring a very low level of sensitivity at the receiver, which can be obtained by allowing the Quality of Service (QoS) to be achieved for low levels of Signal-to-Noise Ratio (SNR) [6]. The low-energy consumption requirement results from the necessity to have a long battery life for the devices (up to 10 years). Regarding the physical layer, saving energy is usually done by selecting an energy-efficient modulation or by considering low-complexity operations for data transmissions (thus reducing the consumption of the processing unit). It is also achieved by ensuring a high efficiency for the power amplifier (PA), which is the most power-consuming component of the transmission chain [7-9]. The efficiency of the PA is highly dependent on the 
peak power of the signal [10]. Constant envelope modulations are thus of prime importance, as they allow for large PA efficiency gains.

Narrow-Band IoT (NB-IoT) is a solution partly dedicated to LPWA networks and was introduced in the LTE release 13 [11-14]. The uplink transmission of this solution combines [13 15] Turbo Code (TC) [11, 15] as forward error correction (FEC) and a repetition factor. It guarantees the QoS to be reached for very low levels of SNR, i.e., low levels of sensitivity are obtained. The solution is highly energy efficient and offers a large range of data rates, making it suitable to numerous applications. A solution relying on a turbo receiver, the Turbo-FSK, was introduced in previous literature [6]. It allows for very low levels of sensitivity to be achieved thanks to the use of a combination of orthogonal modulation, a convolutional code, and an iterative receiver. Additionally, the scheme employs a constant envelope modulation, the Frequency Shift Keying (FSK) modulation, potentially increasing the efficiency of the PA.

If the Turbo-FSK scheme can be easily integrated in an OFDM framework thanks to its use of the FSK (which can be implemented using the fast Fourier transform (FFT) algorithm), it nonetheless lacks the flexibility offered by the NB-IoT solution. An interesting option is therefore to associate this technique with alphabet hybridation [16-19], which will improve the flexibility of the scheme. This hybridation of the FSK modulation, so-called Coplanar FSK [20], can be done while maintaining the constant envelope property, which gives a significant advantage to the Turbo-FSK technique. Additionally, the absence of constraints on the choice of the orthogonal alphabet for Turbo-FSK enables the design of alternative solutions relying on different modulations. For example, the chirp modulation [21] gained significant interest in recent years [22], particularly with LPWA industrial solution LoRa [23-25]. The alphabet of this orthogonal modulation is constructed from a chirp base, which can be, for example, selected as a Zadoff-Chu (ZC) sequence [26], a Constant Amplitude Zero Auto-Correlation (CAZAC) sequence notably used for the primary synchronization signal in the LTE protocol [11]. A possible alternative to Turbo-FSK can thus be the Turbo-ZC scheme, where a chirp modulation is used instead of the FSK modulation.

There are three main objectives and contributions for this work: present the possibility to integrate the TurboFSK in an OFDM framework, confront the scheme to more practical scenarios, and study the potential of the extensions of the scheme. Regarding the first objective, a thorough description of the OFDM framework is proposed, and equations of the several receivers are derived for the specific architecture. For the second objective, several transmission channels are considered: an additive white Gaussian noise (AWGN) channel, a static Rayleigh fading channel, and a Rayleigh fading channel with mobility. Finally, regarding the third objective, two variations of the initial Turbo-FSK scheme are considered: Coplanar Turbo-FSK, which relies on a hybrid FSK and Phase Shift Keying (PSK) alphabet, and Turbo-ZC (and Coplanar Turbo-ZC), which uses an orthogonal alphabet constructed from $\mathrm{ZC}$ sequences. For all the comparisons, two scenarios with two different data rates are considered. Three other schemes, based on the [13 15] TC, are used for comparisons: Turbo Coded Orthogonal Frequency Division Multiplexing (TC-OFDM), Turbo Coded Single Carrier Frequency Division Multiple Access (TCSC-FDMA), and Turbo Coded Frequency Shift Keying (TC-FSK). The first two schemes are directly derived from the NB-IoT solution, while the third one enables a comparison of Turbo-FSK with another turbo coded constant envelope waveform.

The paper is organized as follows. The system model, including the OFDM framework and the five compared solutions, is presented in Section 2. Performance are compared in Section 3, and Section 4 concludes the paper.

\section{System model}

This section is dedicated to the presentation of the general OFDM framework and of the various techniques that are evaluated and compared.

\subsection{OFDM framework}

The general principle of the OFDM framework is to construct the signal in the frequency domain. Over the $N_{\text {FFT }}$ available carrier frequencies, only $N_{A}$ are considered to transmit information. This is a form of multicarrier modulation. The sequence generation in the OFDM framework, i.e., the transmitter architecture, and the considered channel are described first. Then, assuming a generic form for the transmitted sequence, the mathematical derivations of the likelihood of a sequence are computed. The result will be used in the next sections to derive the probabilistic receiver of the considered schemes. The generic architecture of the OFDM framework is finally presented, along with the values of the parameters considered for the simulations.

\subsubsection{Transmitter}

The OFDM framework is depicted in Fig. 1. From the information packet of size $Q$ bits, a cyclic redundancy check (CRC) is computed and appended to the information block. Encoding and modulation steps are then performed. These steps depend on the choice of the technique and are presented later. The output of this block is represented by a matrix of size $N_{A} \times N_{s}$, where $N_{A}$ is the number of active frequencies and $N_{s}$ the number of time slots (or OFDM symbols). This is the time-frequency allocation which is then included in the FFT grid of size 


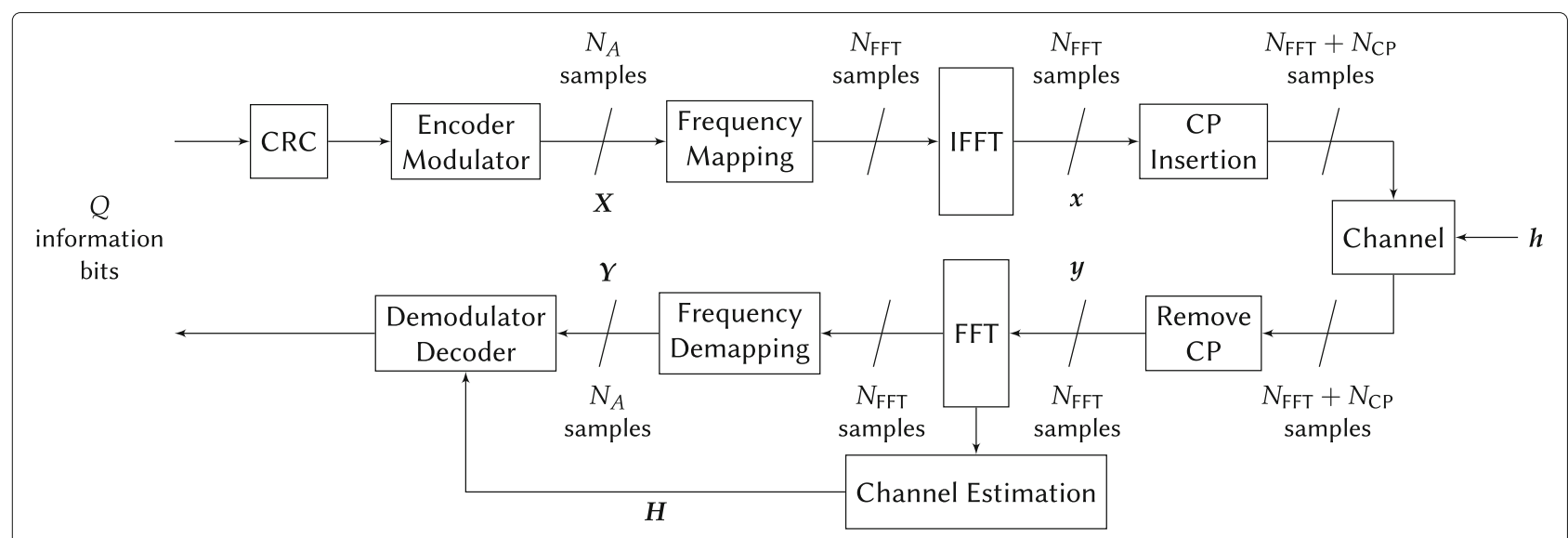

Fig. 1 System model for the OFDM framework

$N_{\mathrm{FFT}} \times N_{s}$. Inverse discrete Fourier transform (IDFT) of size $N_{\text {FFT }}$ is then applied to convert the frequency signal into a time signal consisting of $N_{\mathrm{FFT}} \times N_{s}$ samples (or chips). The inverse fast Fourier transform (IFFT) algorithm is used in practice to perform the IDFT. Considering the vector $\boldsymbol{X}$ of length $N_{\mathrm{FFT}}$, the discrete-time sequence of the considered OFDM symbol $\boldsymbol{x}$ is given by

$$
x(k)=\frac{1}{N_{\mathrm{FFT}}} \sum_{n=0}^{N_{\mathrm{FFT}-1}} X(n) e^{j 2 \pi \frac{k n}{N_{\mathrm{FFT}}},}
$$

which is the IDFT of the vector $X$, with $k \in\left\{0, \ldots, N_{\mathrm{FFT}}-1\right\}$. A cyclic prefix (CP) of length $N_{\mathrm{CP}}$ consisting of the last $N_{\text {CP }}$ samples of each OFDM symbol is then inserted at the beginning of each symbol.

\subsubsection{Channel}

The emitted signal then transits through the channel. The use of a CP allows the effect of the channel to be expressed by a circular convolution when the channel delay spread is smaller than the size of the CP. Multi-path channel is modelled by a discrete-time domain representation, i.e., a finite impulse response (FIR) filter, applied at chip-time. The useful signal (after removing the $\mathrm{CP}$ ) at index $k$ is given by

$$
y(k)=\sum_{l=0}^{L_{\mathrm{ch}}-1} h(l) x(k-l)+v(k),
$$

where $\boldsymbol{h}$ is the channel impulse response, a complex vector with $L_{\mathrm{ch}}$ elements, representing the gain of each possible paths. The elements $v_{k}$ of the noise vector $v$ are independent and follow a circularly symmetric and zero mean complex normal distribution with variance $\sigma_{\mathrm{ch}}^{2}$. The circular convolution assumption can only be considered if $N_{\mathrm{CP}} \geq L_{\mathrm{ch}}$, i.e., if the delays of the previous OFDM symbols only affect the CP of the current symbol and not its useful part.

\subsubsection{Likelihood computation}

In order to derive the probabilistic receiver for each technique, we would like to express the likelihood of observing a sequence $\boldsymbol{y}$ given that a sequence $\boldsymbol{x}$ was transmitted. A generic form for the transmitted sequence is considered, and further simplifications will occur when specific modulation techniques are considered. The likelihood of observing a sequence $\boldsymbol{y}$ given that a sequence $\boldsymbol{x}$ was transmitted is given by

$$
p(\boldsymbol{y} \mid \boldsymbol{x})=\prod_{k=0}^{N_{\mathrm{FFT}}-1} p(y(k) \mid x(k)) .
$$

As normal distribution with variance $\sigma_{\mathrm{ch}}^{2}$ was assumed, the likelihood is expressed

$$
\begin{aligned}
p(\boldsymbol{y} \mid \boldsymbol{x})= & C_{\mathrm{st}} \exp \left\{-\frac{1}{2 \sigma_{\mathrm{ch}}^{2}} \sum_{k=0}^{N_{\mathrm{FFT}}-1}\left|\sum_{l=0}^{L_{\mathrm{ch}}-1} h(l) x(k-l)\right|^{2}\right. \\
& \left.+\frac{1}{\sigma_{\mathrm{ch}}^{2}} \sum_{k=0}^{N_{\mathrm{FFT}}-1}\left\langle y(k), \sum_{l=0}^{L_{\mathrm{ch}}-1} h(l) x(k-l)\right\rangle\right\},
\end{aligned}
$$

where $C_{\mathrm{st}}$ is a term independent of $\boldsymbol{x}$ and $\langle\cdot, \cdot \cdot\rangle$ is the scalar product operator.

Using (1) in (4), the likelihood can be expressed with

$$
\begin{aligned}
p(\boldsymbol{y} \mid \boldsymbol{x})= & C_{\mathrm{st}} \exp \left\{-\frac{1}{2 \sigma_{\mathrm{ch}}^{2}} \frac{1}{N_{\mathrm{FFT}}^{2}} \sum_{k=0}^{N_{\mathrm{FFT}}-1}\left|\sum_{n=0}^{N_{\mathrm{FFT}}-1} X(n) H(n) e^{j 2 \pi \frac{n k}{N_{\mathrm{FFT}}}}\right|^{2}\right. \\
& \left.+\frac{1}{\sigma_{\mathrm{ch}}^{2}} \frac{1}{N_{\mathrm{FFT}}} \operatorname{Re}\left(\sum_{n=0}^{N_{\mathrm{FFT}}-1} \overline{X(n) H(n)} Y(n)\right)\right\}
\end{aligned}
$$

where $Y(n)$ (resp. $H(n))$ is the $n$th dimension of the discrete Fourier transform (DFT) of size $N_{\text {FFT }}$ of the vector $y$ (resp. $\boldsymbol{h}$ ) and $\bar{X}$ is the complex conjugate of $X$. 
As the form of $\boldsymbol{x}$ was purposely kept general, additional computations are required to obtain the final expression of the maximum likelihood (ML) decoder. However, the expression demonstrates the necessity for the receiver to evaluate the DFT of the received time-vector $\boldsymbol{y}$ denoted by $\boldsymbol{Y}$ and to have knowledge of $\boldsymbol{H}$, the DFT of the channel coefficients.

The log likelihood ratio (LLR) of the bit $b_{m}$ is given by

$$
L\left(b_{m}\right)=\log \frac{p\left(\boldsymbol{y} \mid b_{m}=+1\right)}{p\left(\boldsymbol{y} \mid b_{m}=-1\right)}=\log \frac{\sum_{i \in \mathcal{X}_{+1}^{m}} p\left(\boldsymbol{y} \mid \boldsymbol{x}^{i}\right)}{\sum_{i \in \mathcal{X}_{-1}^{m}} p\left(\boldsymbol{y} \mid \boldsymbol{x}^{i}\right)},
$$

where $\mathcal{X}_{+1}^{m}\left(\right.$ resp. $\left.\mathcal{X}_{-1}^{m}\right)$ the group of sequences $\boldsymbol{x}$ which encode an information word for which the bit $b_{m}$ is equal to +1 (resp. -1 ). The LLR of the bits for each technique will be computed using the specific expression of the sequence $\boldsymbol{x}$ in (5) and (6).

\subsubsection{Receiver}

In order to retrieve the information that was mapped in the frequency domain at the transmitter side, a DFT of size $N_{\text {FFT }}$ is applied on each OFDM symbol, using the FFT algorithm. The result is the vector $\boldsymbol{Y}$, the channel observation. By selecting only the $N_{A}$ used frequencies, the $N_{S}$ vectors of size $N_{A}$ transmitted at the first place are retrieved. This signal is fed to the demodulator/decoder along with $\boldsymbol{H}$. This vector can also be interpreted as the complex gain on each frequency. Using the channel observation and the vector $\boldsymbol{H}$, the block demodulator uses a simplified formulation of (5) to compute the LLR of the transmitted bits. The information bits are finally estimated by the decoder and the CRC is computed to evaluate if the packet contains any error.

\subsection{TC-OFDM and TC-SC-FDMA}

Two well-known techniques are considered for comparison: the use of OFDM associated with the [13 15] TC [27] and the TC-SC-FDMA, for which a pre-coding based on a DFT transform is applied. These schemes are multiplexing techniques, i.e., a Quadrature Phase Shift Keying (QPSK) symbol is mapped on each frequency carrier. For both techniques, the encoder/modulator block of Fig. 1 uses a TC encoder and a QPSK modulator. However, since there are $N_{A} N_{s}$ available symbols, a rate matching mechanism is used to repeat or puncture some bits. The rate $R_{m}$ corresponds to the ratio between the number of input bits $(3 Q+12)$ of the rate matcher and the number of output bits $\left(2 N_{A} N_{s}\right)$. If $R_{m}>1$, then bits are punctured (with interleaving to ensure that the punctured bits are evenly spread over time), and when $R_{m}<1$, bits are repeated. An interleaver is used between the encoding and modulation processes. For the case of TC-SC-FDMA, pre-coding using a DFT of size $N_{\text {DFT }}$ is applied.
While the derivations of the receivers could be obtained using the general expression of the likelihood (5), these computations are well known from the literature [28] and are not detailed here. For TC-SC-FDMA technique, the ML receiver is too complex to be considered as likelihood computation involved the whole symbol linked with the precoding. Zero-Forcing (ZF) equalizer is used instead. For the turbo decoder, the max-log approximation is considered and CRC is used as stop criterion for the iterations of the decoder, with a maximum number of iterations set to 10 .

\subsection{Turbo-FSK and Coplanar Turbo-FSK}

The Turbo-FSK principle was introduced in $[6,20,29]$. The motivation for this scheme was to operate close to the Shannon capacity [30] while using a constant envelope modulation. Turbo-FSK can be implemented using an OFDM transceiver. The technique relies on the use of FSK modulation associated with a convolutional code and an iterative decoder. FSK is a common orthogonal modulation, based on an alphabet of orthogonal waveforms equivalent to pure frequencies. When using the previously introduced OFDM framework, only one of the $N_{A}$ possible carriers is activated every time slot. The information is carried by the index of the activated carrier. The innovative aspect of the Turbo-FSK scheme is in the association of the encoding and modulation processes at the transmitter side. The turbo receiver exploits this association to operate at very low levels of SNR.

The design of the Turbo-FSK technique allows for flexibility in terms of choice of alphabet. In order to increase the spectral efficiency of the technique, a hybrid modulation alphabet mixing FSK modulation (orthogonal component) and PSK modulation (linear component) is considered [16]. The alphabet is constructed using $N_{A}$ subsets $\mathcal{A}^{\delta}$, with $\delta \in\left\{0, \ldots, N_{A}-1\right\}\left(N_{A}\right.$ represents the number of possible frequencies for the FSK part of the alphabet), and $M_{L}$ linear shifts given by the complex number $z_{p}=\exp \left\{j 2 \pi p / M_{L}\right\}$, with $p \in\left\{0, \ldots, M_{L}-1\right\}\left(z_{p}\right.$ thus represents the phase shifts from the PSK part of the alphabet). Symbols belonging to the same orthogonal subset are coplanar (they only differ by a complex multiplicative factor), hence the denomination Coplanar Turbo-FSK. A time symbol $x^{i}$ from the alphabet is defined as

$$
\boldsymbol{x}^{i}=\boldsymbol{f}^{\delta} z_{p},
$$

where $\boldsymbol{f}^{\delta}$ is the vector of size $N_{A}$ generating the orthogonal subset $\mathcal{A}^{\delta}$, with

$$
f^{\delta}(k)=\exp \left\{j 2 \pi \frac{\delta k}{N_{\mathrm{FFT}}}\right\},
$$

and $k \in\left\{0, \ldots, N_{\mathrm{FFT}}-1\right\}$. Each orthogonal subset is of size $M_{L}$ and the alphabet size is given by the product $M=$ 
$N_{A} M_{L}$. The values $M, M_{L}$, and $N_{A}$ must be powers of two. The spectral efficiency of the modulation only is given by

$$
\eta_{\text {mod }}=\frac{\log _{2}(M)}{N_{\perp}}=\frac{\log _{2}(M)}{M} \cdot M_{L} .
$$

When setting $M_{L}=1$, the modulation becomes equivalent to a classic FSK modulation with $M$ tones. Dealing with large values of size of FSK modulation leads to low spectral efficiencies [31]. However, for $M_{L}>1$, the phase of the symbols carries information. For a constant value of $M$, increasing the value of $M_{L}$ leads to a reduction of the number of required orthogonal dimensions, thus improving the spectral efficiency of the modulation. Alternatively, for a constant number of orthogonal dimensions (i.e., a constant number of active carriers), increasing the value of $M_{L}$ increases the size of the alphabet and the spectral efficiency.

The encoder/modulator block for the Turbo-FSK is depicted in Fig. 2. Similar to the transmitter presented in [6], the encoder consists in $\lambda$ stages where each stage encodes an interleaved version of the $Q$ information bits. The information block is divided into $N_{q}$ information words of $q$ bits. Eventually, a total of $\lambda \times\left(N_{q}+1\right)$ binary words of length $q+1$ are generated. Each binary word is mapped on one of the codewords of the alphabet. In order to fit the $N_{s}$ available symbols, the rate matcher punctures or repeats some binary words so that

$$
R_{m}=\frac{\lambda \cdot\left(N_{q}+1\right)}{N_{s}} .
$$

Unlike the other schemes, the rate matcher operates at the word level instead of the bit level. This is because entire words of size $q+1$ are associated to one of the symbols from the alphabet. The alphabet size is $M=2^{q+1}$. If the binary word $\boldsymbol{b}$ is associated to the symbol $\boldsymbol{x}^{i}$ as expressed in (7), then the output of the modulator is

$$
X(n)= \begin{cases}z_{p} & \text { if } n=\delta \\ 0 & \text { otherwise. }\end{cases}
$$

This is very similar to FSK signaling as only one frequency carries information. However, unlike FSK where all the information is on the carrier index, additional bits can be mapped on the phase shift of the active carrier. When $M_{L}=1$, the scheme is equivalent to Turbo-FSK, but when $M_{L} \geq 2$, the scheme is called Coplanar TurboFSK. The resulting matrix of size $N_{A} \times N_{S}$ is sparse, as only $N_{s}$ elements are different from 0 .

The demodulator/decoder block is depicted in Fig. 2. First, the likelihood of all the symbols from the alphabet is computed. When combining the general expression (5) with (11), the likelihood given that the symbol $\boldsymbol{x}^{i}$ was sent is expressed

$$
\begin{aligned}
p\left(\boldsymbol{y} \mid \boldsymbol{x}^{i}\right)= & C_{\mathrm{st}} \exp \left\{\frac { 1 } { \sigma _ { \mathrm { ch } } ^ { 2 } N _ { \mathrm { FFT } } } \left(\operatorname{Re}\left(\overline{z_{p} H(\delta)} Y(\delta)\right)\right.\right. \\
& \left.\left.-\frac{1}{2}|H(\delta)|^{2}\left|z_{p}\right|^{2}\right)\right\} .
\end{aligned}
$$

After computing the likelihood of each sequence, a matrix of size $M \times N_{s}$ is obtained, containing the likelihoods of all the possible symbols from the alphabet. The puncturing management block is then in charge to recover the original $M \times \lambda\left(N_{q}+1\right)$ matrix generated at the transmitter. If a symbol was repeated, columns corresponding to the repetitions of this symbol are added. If a symbol was punctured, a column of $0 \mathrm{~s}$ is added. The resulting matrix corresponds to the channel observation of all stages.

The turbo decoder then uses the observation of the $\lambda$ stages to perform the iterative decoding presented in [6] (detailed in [20,29]), using the max-log approximation. The CRC can be used as a stop criteria, as it is computed at the end of each iteration.

\subsection{Turbo-ZC and Coplanar Turbo-ZC}

We propose to extend the Turbo-FSK technique to another family of orthogonal alphabets, the chirp modulation [21, 22]. The chirps are selected as ZC sequences

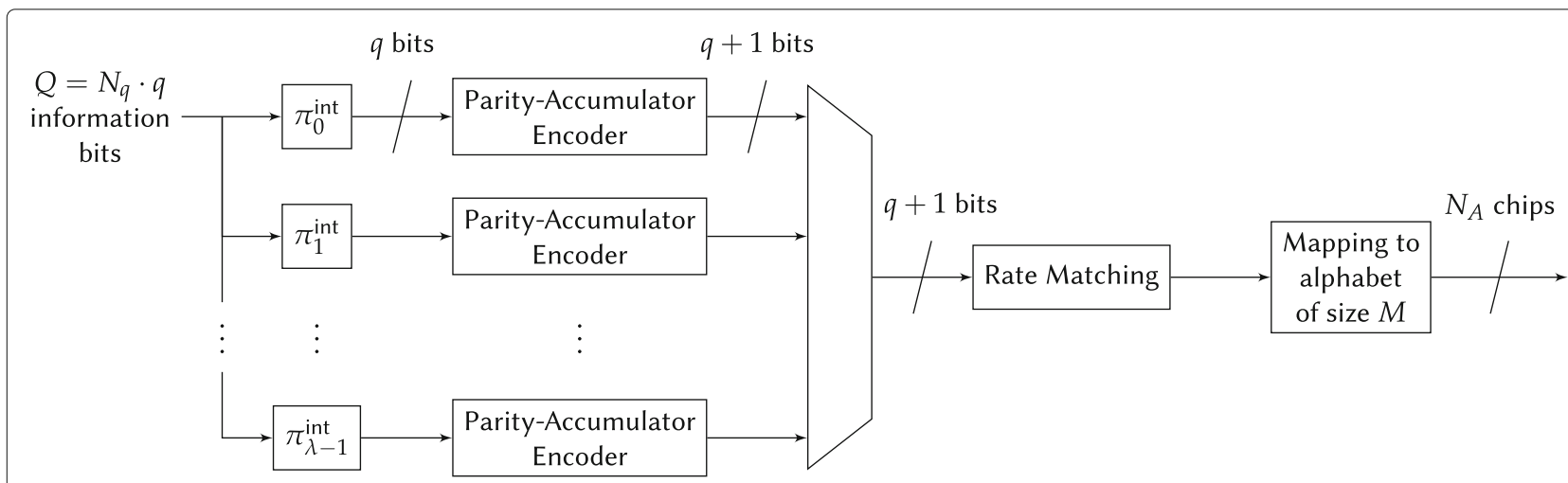

Fig. 2 Encoder/modulator of the Turbo-FSK system 
[26]. In the time expression of the alphabet symbols given in Eq. (7), the vectors $\boldsymbol{f}^{\delta}$ are now constructed using ZC sequences (instead of the pure-tone complex exponential sequences for the Turbo-FSK). However, unlike the Coplanar Turbo-FSK, where symbols of the alphabet are expressed in time, we wish to express the symbols of the Coplanar Turbo-ZC alphabet in frequency. We consider the base ZC sequence $\boldsymbol{g}_{\mu}$ which has two parameters, its length $N_{\mathrm{ZC}}$ and its index $\mu$, both positive integers. The sequence is given by

$$
g_{\mu}(n)=\exp \left\{j \pi \frac{\mu}{N_{\mathrm{ZC}}} n\left(n+\bmod \left(N_{\mathrm{ZC}}, 2\right)\right)\right\},
$$

where $\bmod (\cdot, 2)$ is the modulo two operator, and with $n \in\left\{0, \ldots, N_{\mathrm{ZC}}-1\right\}$. The zero-autocorrelation property of the $\mathrm{ZC}$ sequence allows for the construction of an orthogonal alphabet using circularly shifted versions of a base sequence. The base sequence $\boldsymbol{g}_{\mu}$ with parameters $N_{\mathrm{ZC}}=N_{A}$ and $\mu=1$ is selected.

An interesting implementation approach for the alphabet construction is the combination of the DFT matrix with the base sequence. The vectors $\boldsymbol{f}^{\delta}$ are constructed from vectors of the DFT matrix multiplied by the base sequence $g_{1}$. Its samples are given by

$$
f^{\delta}(n)=g_{1}(n) \exp \left\{j 2 \pi \frac{\delta n}{N_{A}}\right\}=\exp \left\{j \pi \frac{n^{2}+2 \delta n}{N_{A}}\right\},
$$

with $N_{A}$ a power of 2 . This different approach also generates $\mathrm{ZC}$ sequences and allows for an efficient receiver architecture, as presented later.

The encoder/modulator of the Coplanar Turbo-ZC system is strictly equivalent to the one depicted in Fig. 2 for the Turbo-FSK, apart from the modulation alphabet (the vectors generating the orthogonal subsets are expressed with (14) instead of (8)). The symbols from the alphabet will be mapped in the frequency domain, before the IFFT. The output of the block assuming that the current binary word is associated to the $i$ th element of the alphabet is given by

$$
\begin{aligned}
X(n) & =z_{p} g_{1}(n) \exp \left\{j 2 \pi \frac{\delta n}{N_{A}}\right\} \\
& =\exp \left\{j \pi\left(\frac{n^{2}+2 \delta n}{N_{A}}+\frac{2 p}{M_{L}}\right)\right\} .
\end{aligned}
$$

with $n \in\left\{0, \ldots, N_{A}-1\right\}, \delta$ the index for the orthogonal part, and $p$ for the linear part (the PSK alphabet). When $M_{L}=1$, the denomination Coplanar Turbo-ZC is simplified to Turbo-ZC, as each orthogonal subset contains only one symbol.
Unlike the Coplanar Turbo-FSK, the output matrix, of size $N_{A} \times N_{s}$, is no longer sparse. Each column consists of a delayed $\mathrm{ZC}$ sequence. One property of the $\mathrm{ZC}$ sequence is its invariance to FFT operations. However, in this case, the size of the IFFT $N_{\text {FFT }}$ is different from the size of the sequence $N_{A}$; thus, the resulting time signal will not be a CAZAC sequence.

The demodulator/receiver for Coplanar Turbo-ZC is very similar to the one proposed for Coplanar Turbo-FSK, depicted in Fig. 3. The only difference is the likelihood computation. With the expression Eq. (5) obtained from a generic sequence and using Eq. (15), the likelihood is given by

$$
\begin{aligned}
p\left(\boldsymbol{y} \mid \boldsymbol{x}^{i}\right)= & C_{\mathrm{st}} \exp \left\{\frac{1}{\sigma_{\mathrm{ch}}^{2}} \frac{1}{N_{\mathrm{FFT}}}\right. \\
& \left.\times \operatorname{Re}\left(\overline{z_{p}} \sum_{n=0}^{N_{A}-1} \overline{g_{1}(n) H(n)} Y(n) e^{-j 2 \pi n \delta / N_{A}}\right)\right\} .
\end{aligned}
$$

Note that the first part of Eq. (5) is included in the constant, as it can be shown independent of the index $i$. In order to compute the likelihood, each received sequence of size $N_{A}$ is multiplied by the conjugate of the channel coefficients and by the conjugate of the base sequence $g_{1}$ used to build the alphabet. A FFT of size $N_{A}$ is then applied. The likelihood of the codeword $i$ is finally obtained by multiplying the $\delta$ th output of the FFT by the linear coefficient $p$ and retrieving the real part. Compared to the Coplanar Turbo-FSK, the computation of the likelihood for the Coplanar Turbo-ZC requires two additional steps: the multiplication by the base sequence and the FFT of the size $N_{A}$.

\subsection{TC-FSK}

The use of the FSK modulation associated with the [13 15] TC can also be considered. For this scheme, the transmitter performs turbo encoding associated with a rate matching mechanism and an interleaver. FSK modulation is applied and the output is expressed with (11). The receiver computes the LLR of the binary sequence using

$$
\begin{aligned}
L\left(b_{m}\right)= & \max _{i \in \mathcal{X}_{1}^{m}}\left[\operatorname{Re}(\overline{H(i)} Y(i))-\frac{1}{2}|H(i)|^{2}\right] \\
& -\max _{i \in \mathcal{X}_{-1}^{m}}\left[\operatorname{Re}(\overline{H(i)} Y(i))-\frac{1}{2}|H(i)|^{2}\right],
\end{aligned}
$$

which is obtained by combining Eqs. (12) and (6) and the max-log approximation. Turbo decoding is then performed, similar to the TC-OFDM. Unlike Coplanar Turbo-FSK, the TC-FSK scheme does not combine the demodulation and the decoding in a joint process. It nonetheless offers a constant envelope modulation. 


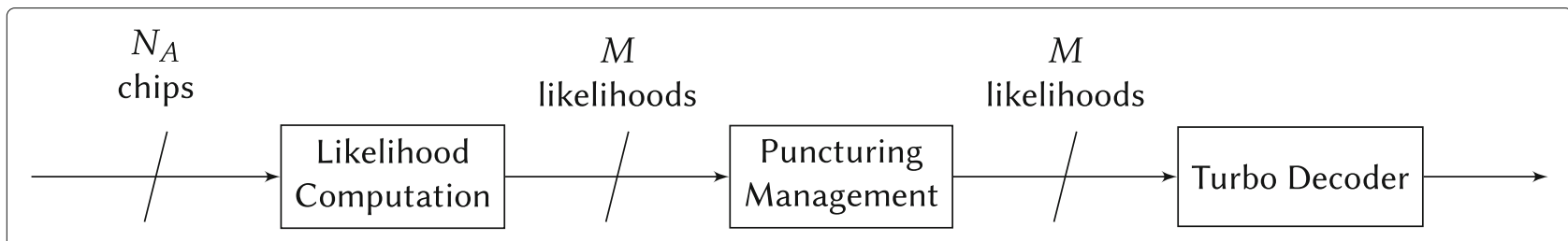

Fig. 3 Demodulation/decoder of the Turbo-FSK system

\section{Results and discussion}

The performance of each scheme is assessed over several types of transmission channels, and the envelope variations of each technique is evaluated. In order to restrict the study, some parameters are kept constant for all the schemes. As all the schemes were presented associated with the same FFT configuration, the parameters for this architecture can be taken equal for all the schemes. The parameters are chosen as corresponding to the mode 1.4 MHz of LTE, i.e., latest NB IoT standard [14]. Two different scenarios are considered: a low-rate $(8.24 \mathrm{kbps})$ as well as a high-rate scenario ( $46.68 \mathrm{kbps})$. This will exhibit the impact of the parameters of the schemes on the performance, notably the size of the linear part of the alphabet for both Coplanar Turbo-FSK and Coplanar Turbo-ZC. Specific values for the parameters of the OFDM framework are selected. The selected parameters are given in Table 1 , where $f_{s}$ is the sampling frequency, $\Delta f$ the subcarrier spacing and $f_{c}$ the carrier frequency. With $N_{A}=16$ active carriers, the bandwidth of the transmitted signal is equal to $B=240 \mathrm{kHz}$. The spectral efficiency can be computed with

$$
\eta=\frac{Q}{N_{s}\left(N_{\mathrm{FFT}}+N_{\mathrm{CP}}\right)}
$$

and the data rate is given by $R=Q / N_{s}$.

The first scenario considers the transmission of $Q=$ 1000 bits over $N_{s}=1700$ OFDM symbols. The spectral efficiency is equal to $\eta=4.29 \cdot 10^{-3} \mathrm{bits} / \mathrm{s} / \mathrm{Hz}$, which gives a data rate of $8.24 \mathrm{kbps}$, a rather low value. This value is consistent with the fact that long-range solutions must transmit at low data rates to provide low sensitivity levels $[6,20]$. Due to the specific number of symbols, the rate matcher of each technique has a different output rate. The equivalent number of repetitions given by $1 / R_{m}$ and the parameters for each technique is presented in Table 2. For this scenario, we consider the Turbo-FSK and Turbo-ZC

Table 1 Parameters for the OFDM framework. The CP duration is equal to $4.6875 \mu \mathrm{s}$

\begin{tabular}{llllll}
\hline$N_{A}$ & $N_{\text {FFT }}$ & $N_{\mathrm{CP}}$ & $f_{S}$ & $\Delta f$ & $f_{C}$ \\
\hline 16 & 128 & 9 & $1.92 \mathrm{MHz}$ & $15 \mathrm{kHz}$ & $2.5 \mathrm{GHz}$ \\
\hline
\end{tabular}

(thus $M_{L}=1$ ) with $\lambda=5$. According to the results found in [32], it is the value of $\lambda$ that gives the lowest $E_{b} / N_{0}$ for this size of alphabet $(M=16)$.

For the second scenario, a higher data rate is considered. The same packet size $M=1000$ is transmitted over $N_{s}=300$ OFDM symbols. This gives a spectral efficiency of $\eta=2.43 \cdot 10^{-2} \mathrm{bits} / \mathrm{s} / \mathrm{Hz}$ and a data rate equal to $46.68 \mathrm{kbps}$. The TC-FSK solution is not considered for this scenario, as the value of $N_{s}$ would imply a very large number of punctured bits, making the solution inadequate. The parameters for the four compared techniques are presented in Table 3. Both Coplanar Turbo-FSK and Coplanar Turbo-ZC are selected with parameters $M_{L}=32$ and $\lambda=$ 3 , which gives an alphabet size equal to $M=512$. In [32], the combination $M=512$ and $\lambda=3$ was demonstrated to minimize the required $E_{b} / N_{0}$, for the orthogonal case (i.e., $M_{L}=1$ ). However, for this specific configuration, a loss in required $E_{b} / N_{0}$ is expected for two reasons: $22 \%$ of the symbols are punctured and the choice of $M_{L}=32$ PSK will reduce the energy efficiency.

The performance of the packet error rate (PER) is simulated for various levels of SNR and three different channels: the AWGN channel and a Rayleigh fading channel with both static and mobile conditions (with classical Jake's model [33]). Perfect time and frequency synchronizations are assumed.

\subsection{Under AWGN}

The AWGN channel, i.e., a channel with a single and constant propagation path, is first considered. For the first scenario, the PER performance of the five different techniques is depicted in Fig. 4a, versus the SNR. Very low levels of SNR are considered. The TC-OFDM and TC-SC-FDMA schemes are equivalent under AWGN

Table 2 Parameters for the first scenario. The data rate is equal to $8.24 \mathrm{kbps}$

\begin{tabular}{llllll}
\hline$N_{A}$ & 16 & $N_{S}$ & 1700 & $\eta(\mathrm{bits} / \mathrm{s} / \mathrm{Hz})$ & $4.29 \cdot 10^{-3}$ \\
\hline Waveforms & TC-OFDM & TC-SC-FDMA & TC-FSK & Turbo-ZC & Turbo-FSK \\
Modulation & QPSK & QPSK & FSK & $\begin{array}{l}M_{L}=1, \\
\lambda=5\end{array}$ & $M_{L}=1$, \\
& & & & $\lambda=5$ \\
$1 / R_{m}$ & 17.76 & 17.76 & 2.22 & 1 & 1 \\
\hline
\end{tabular}


Table 3 Parameters for the second scenario. The data rate is equal to $46.68 \mathrm{kbps}$

\begin{tabular}{|c|c|c|c|c|c|}
\hline$N_{A}$ & 16 & $N_{S}$ & 300 & $\begin{array}{l}\eta \\
\text { (bits/s/Hz) }\end{array}$ & $2.43 \cdot 10^{-2}$ \\
\hline Waveforms & TC-OFDM & TC-SC-FDMA & $\begin{array}{l}\text { Coplanar } \\
\text { Turbo-ZC }\end{array}$ & $\begin{array}{l}\text { Coplanar } \\
\text { Turbo-FSK }\end{array}$ & \\
\hline Modulation & QPSK & QPSK & $\begin{array}{l}M_{L}=32 \\
\lambda=3\end{array}$ & $\begin{array}{l}M_{L}=32 \\
\lambda=3\end{array}$ & \\
\hline $1 / R_{m}$ & 3.14 & 3.14 & 0.78 & 0.78 & \\
\hline
\end{tabular}

and offer the best performance. Turbo-FSK and Turbo$\mathrm{ZC}$ are also equivalent under this channel and exhibit a 0.2- $\mathrm{dB}$ loss at a PER of $10^{-3}$. Better performance could be achieved by selecting other combinations of alphabet size and number of repetition $\lambda$ [32]. The TC-FSK suffers from a loss of $2.9 \mathrm{~dB}$ for the same PER versus TC-OFDM. Unlike Turbo-FSK, the receiver of this scheme does not combine FSK demodulation and turbo decoding in the same procedure. Better performance is achieved using a more sophisticated receiver such as the one considered in Turbo-FSK.

The PER performance for the second scenario and for the four compared techniques is depicted in Fig. 4b. The levels of required SNR are higher than those for the first scenario, due to the increase of the data rate. Similar to the first scenario and due to the choice of the AWGN channel, TC-OFDM and TC-SC-FDMA are equivalent for this scenario, as well as Coplanar Turbo-FSK and Coplanar Turbo-ZC. Their performance is less than $0.4 \mathrm{~dB}$ away from the TC-OFDM, for a PER of $10^{-2}$. With this size of alphabet and number of repetitions $(M=512$ and $\lambda=3$ ), the Turbo-FSK (i.e., with $M_{L}=1$ ) overcomes the [13 15] TC [20]. However, the use of the linear modulation of size 32 associated with the puncturing of $22 \%$ of the codewords induces a loss of performance.
These simulations include the loss in spectral efficiency incurred by the introduction of the $\mathrm{CP}$ and the use of the CRC. The first is necessary to avoid inter-symbol interference (ISI) and inter-carrier interference (ICI) when considering multi-path channels. The latter is used to detect errors in the decoded packet and to compute the PER. Since these two elements induce a loss in spectral efficiency, this corresponds to a loss in $E_{b} / N_{0}$. Using a CRC of size 16, the loss is expressed in decibel as

$$
10 \log _{10} \frac{N_{\text {FFT }}}{N_{\text {FFT }}+N_{\mathrm{CP}}}+10 \log _{10} \frac{Q}{Q+16} .
$$

With the considered parameters, this loss is equal to $0.37 \mathrm{~dB}$. These results demonstrate the benefits of considering a joint coding and modulation scheme with respect to the use of FSK and a classical turbo code. When compared to turbo-coded modulations with repetition scheme, the performance loss of the proposed scheme is less than a few tenth of a decibel.

\subsection{Under static Rayleigh fading channel}

Simulations under the static Rayleigh fading channel are considered. Static implies that there is no relative velocity between the transmitter and the receiver, i.e., the channel does not evolve during the transmission of the packet. The Extended Typical Urban (ETU) profile [34, p. 191] is considered for the path gains and delays. This profile has a channel delay spread equal to $\tau=5 \mu \mathrm{s}$, while the $\mathrm{CP}$ duration is equal to $4.6875 \mu \mathrm{s}$. This profile thus represents difficult conditions for the OFDM system. The coherence bandwidth of the channel is equal to $\tau=1 / \tau=200 \mathrm{kHz}$. Since the bandwidth of the signal $B=240 \mathrm{kHz}$ is superior to the coherence bandwidth, frequency diversity is guaranteed.

In order to compare the schemes, the performance over a large number of channel realizations is computed (each realization is randomly generated following the Rayleigh
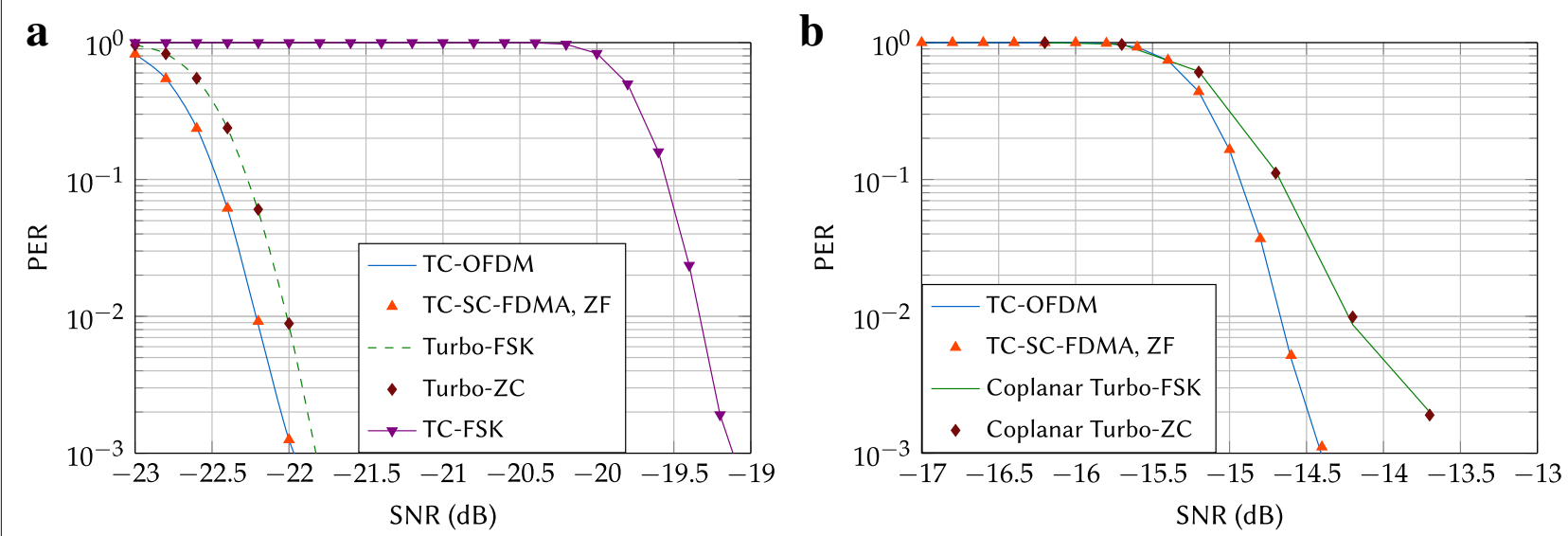

Fig. 4 Performance of the compared solutions under AWGN, for the first scenario (a) and for the second scenario (b) 
model). Average performance over the channel realizations is presented. Since each realization has its own gain, the SNR is estimated at the input of the receiver. In the figures, the average SNR over all the realizations is presented on the horizontal axis. All the compared techniques are stressed with the same realizations of the channel. A large number of realizations is computed, and perfect channel state information (CSI) is considered.

The performance of the five schemes for the first scenario is depicted in Fig. 5a. TC-OFDM outperforms all the other schemes with a PER of $10^{-2}$ at $-11.4 \mathrm{~dB}$ of SNR. Performance of Turbo-ZC and Turbo-FSK is only 0.1 and $0.2 \mathrm{~dB}$ respectively away from TC-OFDM. TCFSK exhibits a loss of $3.1 \mathrm{~dB}$ with respect to TC-OFDM, as for the AWGN case. The performance loss compared to Turbo-FSK is due to the use of a different decoder, since the signaling technique is identical. TC-SC-FDMA exhibits the worst performance, but this can be incurred to the choice of the ZF equalizer. Other equalizers could be considered such as the minimum mean square error (MMSE) and may lead to better performance.

For the second scenario, the performance of the four schemes is depicted in Fig. 5b. As for the case of the AWGN channel, increasing the data rate also increased the range of SNR for equivalent performance. Similar to the first scenario, the TC-SC-FDMA exhibits poor performance due to the use of the ZF equalizer. TC-OFDM and Coplanar Turbo-ZC have similar performance, reaching a PER of $10^{-2}$ at a SNR of $-3.8 \mathrm{~dB}$. Unlike the other scenario, Coplanar Turbo-FSK suffers from a 1.8-dB loss compared to TC-OFDM and Coplanar Turbo-ZC for the same PER. Both Coplanar Turbo-FSK and Coplanar Turbo-ZC use the exact same parameters. The difference in performance can thus be attributed to the use of different signaling techniques. Since the ZC signaling spreads the energy of one symbol on all the active carriers, frequency diversity is better exploited. Carriers with higher gains may compensate for faded carriers. However, in the FSK case, a fading on the frequency that carries the energy will induce the loss of the symbol. The difference in performance is reduced in the other scenario, which has a lower data rate. This may suggest that the use of a higher order of linear modulation $\left(M_{L}=32\right.$ for this scenario) amplifies the difference between ZC and FSK signaling.

For the low-throughput case, performance remains the same for TC-OFDM system and the proposed scheme (Turbo-FSK and Turbo-ZC). In case of high throughput, the frequency diversity provided by Turbo $\mathrm{ZC}$ gives an advantage with respect to Coplanar Turbo-FSK making Coplanar Turbo-ZC interesting when compared to TC-OFDM.

\subsection{Under Rayleigh fading channel with mobility (Jakes-Doppler spectrum)}

Relative mobility between the transmitter and the receiver is now considered, along with the ETU fading profile. The relative velocity between the transmitter and the receiver is equal to $v=50 \mathrm{~km} / \mathrm{h}$. With the selected carrier frequency, given in Table 1 , the Doppler frequency is equal to $f_{d}=115.8 \mathrm{~Hz}$, giving a coherence time of $t_{\mathrm{coh}}=1 / f_{d}=$ $8.63 \mathrm{~ms}$. The coherence is thus 129 times greater than the symbol time (equal to $66.6 \mu \mathrm{s}$ ). The receiver can assume a constant vector $\boldsymbol{H}$ for each symbol, which is actually the time-average estimation of the true channel.

For the first scenario, the performance of the four schemes under mobility condition is depicted in Fig. 6a. When compared to the case of the static ETU depicted in Fig. 5, mobility significantly improves the performance of all the schemes. These schemes benefit from a large amount of redundancy (for example, the encoded bits for TC-OFDM and TC-SC-FDMA are repeated more than 17
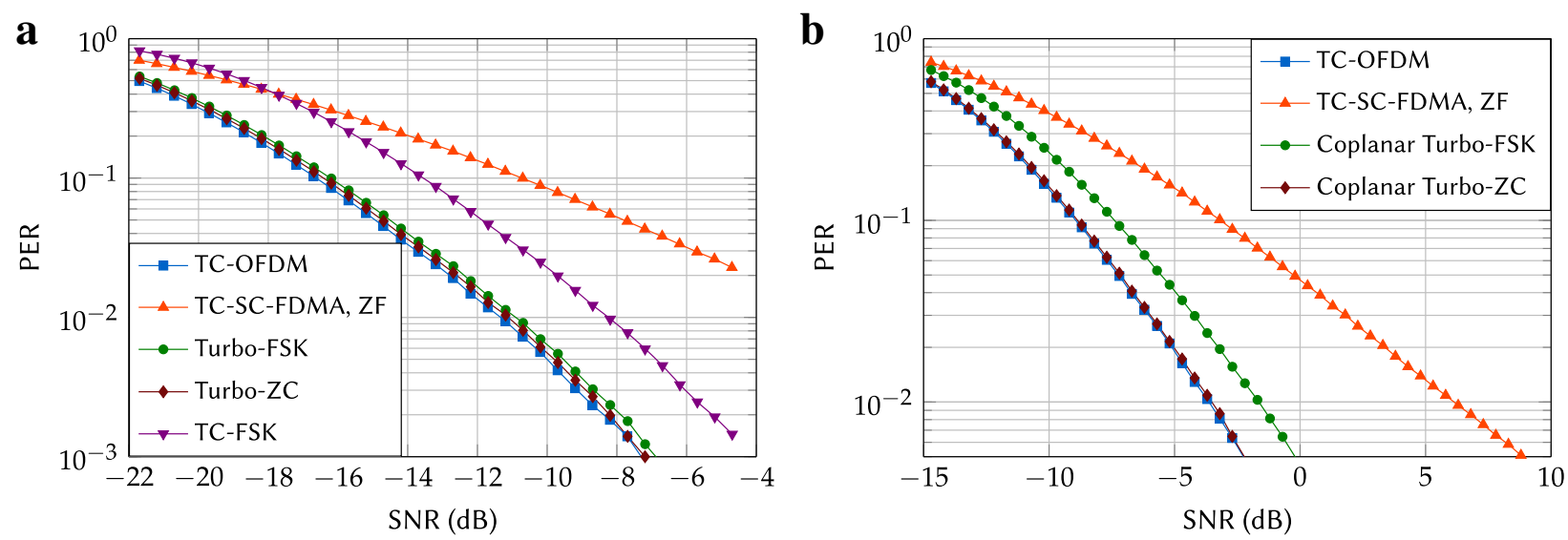

Fig. 5 Performance of the five compared solutions under static Rayleigh fading channel with ETU profile, for the first scenario (a) and for the second scenario (b) 

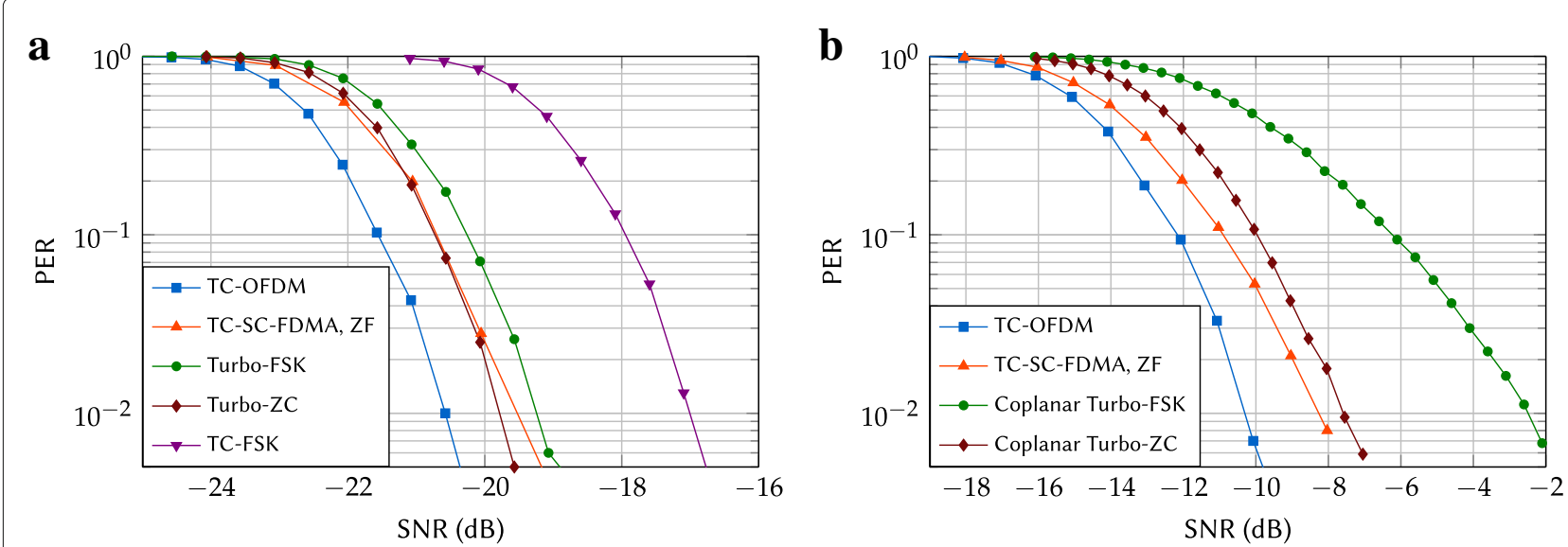

Fig. 6 Performance of the five compared solutions under Rayleigh fading channel with ETU profile. The relative speed is equal to $50 \mathrm{~km} \cdot h^{-1}$ and the carrier frequency is set to $2.5 \mathrm{GHz}$, for the first scenario (a) and for the second scenario (b)

times, and the TC itself offers redundancy). In the mobility case, a symbol and its repetitions experience different channel gains. This is a form of time diversity and performance tends toward the AWGN case when the order of diversity is very large [31]. While the TC-SC-FDMA exhibited poor performance in the static ETU case, the time diversity offered by the repetitions overcomes the negative impact of equalization and the scheme is less than $1 \mathrm{~dB}$ away from TC-OFDM. The TC-FSK scheme is $3.5 \mathrm{~dB}$ away from the TC-OFDM technique. The difference of performance in the static ETU case was in the same order of magnitude. Thanks to the use of repetition and a low spectral efficiency modulation technique, the TC-FSK scheme also benefits from diversity. TurboFSK and Turbo-ZC now exhibits a 0.6 and $0.4-\mathrm{dB}$ gap respectively versus TC-OFDM. Both schemes use $\lambda=5$ repetitions, which offers a diversity comparable to the other techniques.

The performance under mobility conditions for the second scenario is depicted in Fig. 6b. Both the TC-OFDM and TC-SC-FDMA schemes benefit from diversity due to the redundancy but at a smaller extent as there are only three repetitions. The curves tend less towards the AWGN case. While the Coplanar Turbo-ZC reached the performance of TC-OFDM for the static ETU case and was $0.5 \mathrm{~dB}$ away at a PER of $10^{-2}$ in the AWGN case, the gap with TC-OFDM is now equal to $2.4 \mathrm{~dB}$. Coplanar Turbo-FSK exhibits an even worse performance and the gap between Coplanar Turbo-FSK and Coplanar Turbo$\mathrm{ZC}$ is now equal to $5.4 \mathrm{~dB}$. For these two techniques, the lack of diversity gain could be explained by the combination of two aspects: the reduction of the value of $\lambda$ to 3 and the rather high number of punctured codewords. Also, with the configuration $M_{L}=32$, more information is mapped on the linear dimensions. This combination has a devastating effect for the Coplanar Turbo-FSK, but it has less effects on the Coplanar Turbo-ZC, most likely due to its better frequency diversity. The spread spectrum feature of the ZC signaling seems again beneficial, and Coplanar Turbo-ZC outperforms Coplanar Turbo-FSK. When considering an even higher speed, Coplanar Turbo-ZC, Coplanar Turbo-FSK, and TC-FSK will not suffer from ICI since there is no multiplexing (unlike TC-OFDM and TC-SC-FDMA). This will nonetheless induce a spreading of the energy over the adjacent frequencies, i.e., a loss of power of the transmitted frequency for FSK signaling (along with a gain in the adjacent frequencies) and a distortion of the $\mathrm{ZC}$ sequence for the $\mathrm{ZC}$ signaling.

In case of mobility associated with fading channel, TCOFDM remains the best choice. The use of spreading, i.e., SC-FDMA, enables to combine low envelope variation as well as good PER performance. We see here the limitations of the proposed schemes. However, we can expect a similar performance in terms of PER when considering Coplanar Turbo-ZC with respect to SC-FDMA. However, as previously mentioned, the proposed scheme drastically reduces the envelope variation of signal. Therefore, we describe, in the next subsection, a framework to globally compare the different solutions taking into account both channel performance and power consumption.

\subsection{Envelope variations}

In order to study the variations of the envelope, we propose to study the Instantaneous-to-Average Power Ratio (IAPR) [35], which is equivalent to a Peak-to-Average Power Ratio (PAPR) over one sample. The study of the IAPR is more relevant than the measure of the PAPR over a larger number of samples, as it will consider all the samples that potentially reach the non-linear region of the PA [35]. Evaluating the envelope variations is critical to tune the operating point of the PA, the last and most energyconsuming elements of the transmission chain $[7,9]$. The 
efficiency of the PA will influence both the consumption of the system and its cost. Since the IAPR describes the variation of the envelope, it also indicates how to drive the input of the PA in order to avoid the saturation and non-linear region. Usually, a backoff from the saturation point is considered, to ensure the saturation to be reached for only a given probability. However, the consequence of selecting a large back-off (or equally, that high values for the IAPR are more likely) is that the average level of output power is lowered. The efficiency of the PA can be shown to be dependent on the level of output power [10]. Consequently, the variation of the envelope is a major concern regarding the consumption of the system.

For all the compared solutions, the IAPR is measured from the time signal which is sent through the channel, after the addition of the $\mathrm{CP}$ in Fig. 1. For a sufficient statistic, 1000 packets are simulated using the parameters of Table 1.

The Complementary Cumulative Distribution Function (CCDF) of the IAPR is depicted for the five schemes in Fig. 7. TC-OFDM has the largest variations and exhibits a probability of $10^{-3}$ to have an IAPR larger than $8 \mathrm{~dB}$. TCSC-FDMA shows a $2-\mathrm{dB}$ improvement in its variations, thanks to the use of pre-coding. As expected, TC-FSK and Coplanar Turbo-FSK have the same performance, i.e., an IAPR of $0 \mathrm{~dB}$. Both techniques rely on the use of FSK modulation which exhibits a constant envelope, and the Coplanar Turbo-FSK employs phase shifts as for the coplanar value, maintaining the constant envelope property. However, even though the Coplanar Turbo-ZC was constructed using CAZAC sequences, it does not exhibit a constant envelope. This is because the sequence was mapped in the frequency domain. The use of the IFFT oversamples the sequence to give a resulting time signal convolved with the IFFT window that does not have a constant envelope. Additionally, the number of possible sequences is equal to 16 (the value of $N_{A}$ ), i.e., the variations of a given signal will correspond (on average) to the variations of the 16 possible sequences. When the ratio $N_{\mathrm{FFT}} / N_{A}$ is an integer, all the sequences have the same distribution of the IAPR. The IAPR consists in a finite number of values (hence the discrete CCDF) and could be evaluated simply by considering the base sequence. The maximum IAPR of the Coplanar Turbo-ZC appears to be around $2.6 \mathrm{~dB}$, a value that depends on the value of $N_{A}$ and the choice of the base sequence.

The measure of the IAPR clearly emphasizes the main benefits of the proposed schemes, the constant amplitude property of Coplanar Turbo-FSK, and the low PAPR of Coplanar Turbo-ZC, offering a gain of $5.4 \mathrm{~dB}$ versus TCOFDM. It also gives information on how far you must reduce the input power (Input Backoff (IBO)) of a PA in order to limit non-linearity effects that could decrease the performance of the system.

\subsection{Energy consumption}

In the previous section, we stress the gain/loss we have considering new modulation and coding scheme. As a synthesis, we propose to balance the gain/loss through an estimation of the energy consumption of a device. In one hand, the proposed schemes are working worse on channel with mobility. On the other hand, a low PAPR level is achieved. The relation between the IBO and PA efficiency was previously mentioned. The theoretical efficiency of the PA can actually be shown conversely dependent on the level of IAPR [10, 36]. In Fig. 8, the drain efficiency versus the Input Backoff is depicted for class A amplifiers, as well as the corresponding efficiency and PAPR of the five techniques when considering a CCDF of $10^{-3}$ for the IAPR. By selecting Coplanar Turbo-FSK instead of TC-OFDM, the theoretical efficiency of the PA can be increased by $37 \%$. Coplanar Turbo-ZC also offers a gain in efficiency. Regarding the second scenario previously introduced, using the Coplanar Turbo-ZC seems to be an interesting trade-off versus TC-OFDM, as the PA efficiency can be increased while maintaining almost similar performance

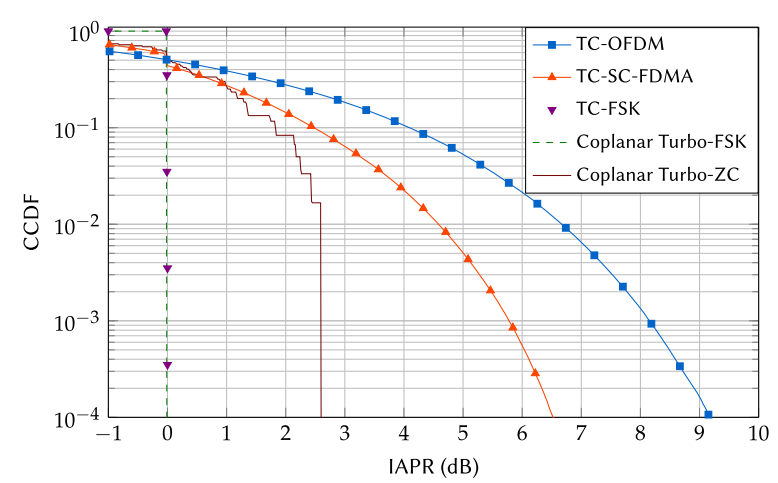

Fig. 7 IAPR of the five compared solutions with the considered OFDM parameters

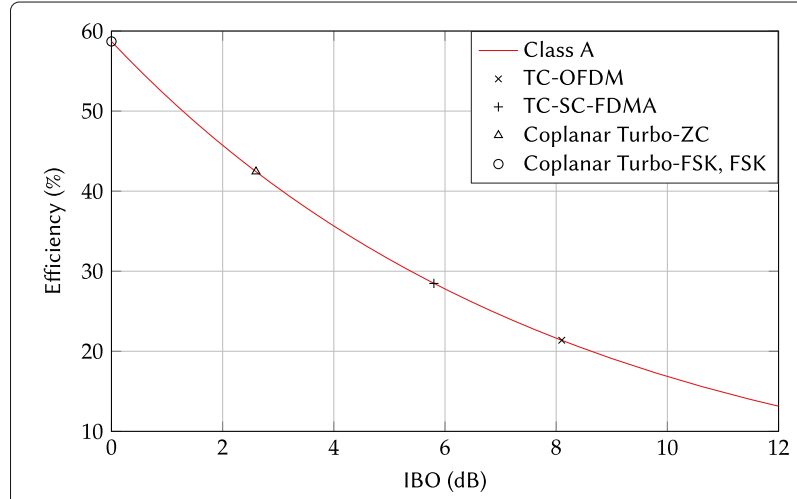

Fig. 8 Drain efficiency versus IBO for class A amplifier, and efficiencies of the five compared solutions for the given scenario 
(unlike the Coplanar Turbo-FSK, which exhibits a more important performance loss). In order to illustrate this effect, we propose to consider the energy consumption associated to one packet.

The energy $E$ consumed by the transmission of the packet can be used to estimate the battery life. It is usually expressed by the product $P_{t} T$, where $P_{t}$ is the transmission power for the given QoS and $T$ the packet duration [9]. However, since the PA has a specific efficiency $\varepsilon<1$ (which depends on the technology used), the consumed energy is actually given by

$$
E=\frac{P_{t} T}{\varepsilon}
$$

where the values of $P_{t}$ and $\varepsilon$ vary depending on the technology selected. For a given QoS, if the required SNR is $\Delta$ $\mathrm{dB}$ lower for the scheme $\mathrm{X}$ than for the considered reference, then the transmitted power can be reduced by $\Delta \mathrm{dB}$. The efficiency $\varepsilon$ is given by the value of the PAPR, which is selected for a CCDF of $10^{-3}$. While considering the TCOFDM as a reference, the ratio of consumed energy is given by

$$
\rho_{\mathrm{X}}=\frac{\varepsilon_{\mathrm{X}}}{\varepsilon_{0} \Delta_{\mathrm{X}}}
$$

where $\varepsilon_{0}$ is the efficiency for the TC-OFDM, $\varepsilon_{\mathrm{X}}$ for the technique $\mathrm{X}$, and $\Delta_{\mathrm{X}}$ the difference in SNR (in linear) between the technique $\mathrm{X}$ and the TC-OFDM. When $\rho_{\mathrm{X}}<$ 1 , this means that compared to TC-OFDM, more energy is consumed, and eventually the battery life is reduced. When $\rho_{\mathrm{X}}>1$, battery life is increased. This quantity describes two variations, the efficiency of the PA and the difference in performance, and how they can potentially balance each other. Indeed, a technique having a very good efficiency but poor performance may have a value of $\rho_{\mathrm{X}}$ inferior to 1 , meaning that the loss in performance is not balanced by the increase of the efficiency.

For all the scenarios and the channels, the ratio of consumed energy is depicted in Fig. 9. TC-SC-FDMA offers a small gain in energy consumption, except for the static ETU case where its low performance induces an increase of the transmitted power. It should be mentioned that better performance could be obtained by considering a different equalizer. TC-FSK offers a gain thanks to its constant envelope. Coplanar Turbo-FSK gives important gains (up to 2.5 under AWGN, i.e., an increase of the battery life by a factor 2.5) but suffers from its low performance under mobility conditions. Finally, Coplanar Turbo-ZC offers an interesting trade-off with gains included between 1 and 1.9 depending on the conditions.

To conclude, both proposed schemes really improve the power consumption with respect to TC-OFDM and SCFDMA. Coplanar Turbo-FSK is particularly interesting for static channel but suffers from poor performances in case of mobility. In the latest case, Coplanar Turbo-ZC offers a tradeoff. When high throughput and mobility are considered, one can expect the best performance with respect to TC-OFDM and SC-FDMA. In case of low throughput, performance of Coplanar Turbo-ZC remains below coplanar Turbo-FSK but the gap is quite small. This makes Coplanar Turbo-ZC a promising scheme.

\section{Conclusions}

In the context of LPWA networks, reaching low levels of sensitivity and reducing the consumption at the device level are major concerns. Nonetheless, coexistence and integration into existing frameworks should be considered to ease the deployment procedure and reduce costs. In this work, we proposed the integration of Turbo-FSK in the OFDM framework and extended the original scheme to the Coplanar Turbo-FSK, which employs a hybrid modulation alphabet, and to the Coplanar Turbo-ZC, which uses another orthogonal alphabet based on $\mathrm{ZC}$ sequences.

We demonstrated the possibility for the proposed techniques to use a transmitter and a receiver based on an OFDM-like architecture. Simulations demonstrated that the proposed schemes achieve performance close

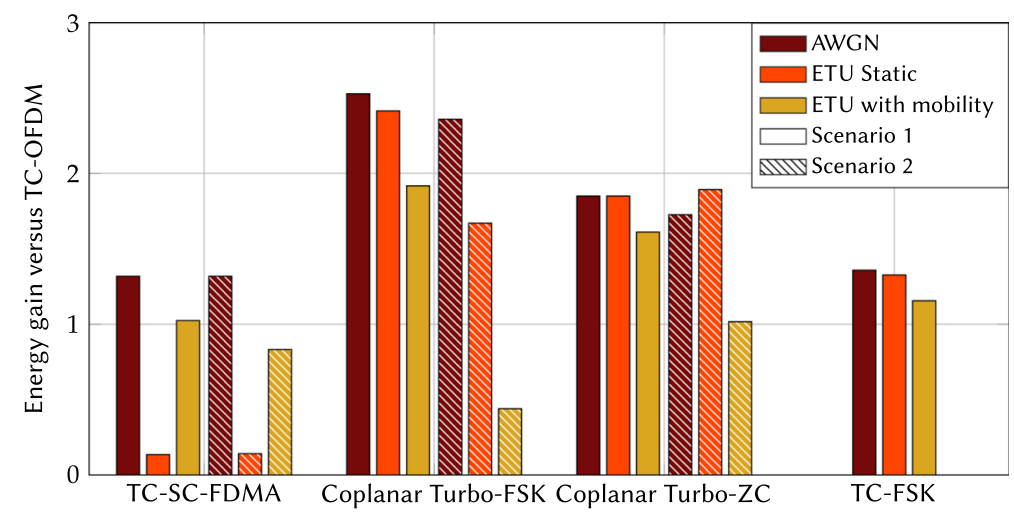

Fig. 9 Energy consumption with respect to the TC-OFDM 
to TC-OFDM in AWGN conditions and Rayleigh fading channels for both static and mobility conditions. However, while TC-OFDM exhibits important envelope variations, a known effect for this modulation, the proposed techniques offer different levels of reduction of the envelope variations, which improves the efficiency of the PA. For example, using Coplanar Turbo-FSK with a class A amplifier can lead to a theoretical drain efficiency of $60 \%$. Coplanar Turbo-FSK trades a small loss in PER for a constant envelope and is a very powerful alternative to the use of classic OFDM, potentially increasing the battery life by a factor 2.5. The presented Coplanar Turbo-ZC exhibits higher envelope variations but exploits the frequency diversity in a better way, offering performance even closer to the TC-OFDM. It appears to be an even more interesting alternative to TC-OFDM and offers a battery life gain for all the considered conditions.

The proposed solutions can be seen as promising additional modes for LPWA transmission, as they can be included in a scheduled network based on an OFDM framework. The solutions could be part of the NB-IoT standard as a new low-power mode which allows a significant reduction of the consumption at the device level.

\section{Abbreviations}

4G: Fourth generation; 5G: Fifth generation; AWGN: Additive white Gaussian noise; CAZAC: Constant amplitude zero auto-correlation; C-TFSK: Coplanar turbo-FSK; CCDF: Complementary cumulative distribution function; CC: Convolutional code; CFO: Carrier frequency offset; CP: Cyclic prefix; CRC: Cyclic redundancy check; CSI: Channel state information; DFT: Discrete Fourier transform; ETU: Extended typical urban; FEC: Forward error correction; FFT: Fast Fourier transform; FIR: Finite impulse response; FSK: Frequency shift keying; IAPR: Instantaneous-to-average power ratio; IBO: Input backoff; ICI: Inter-carrier interference; IDFT: Inverse discrete Fourier transform; IFFT: Inverse fast Fourier transform; loT: Internet of things; ISI: Inter-symbol interference; LLR: Log likelihood ratio; LPWA: Low-power wide-area; LTE: Long-term evolution; ML: Maximum likelihood; MMSE: Minimum mean square error; NB-IoT: Narrow-band loT; NB: Narrow band; OFDM: Orthogonal frequency division multiplexing; PAPR: Peak-to-average power ratio; PA: Power amplifier; PER: Packet error rate; PHY: Physical; PSK: Phase shift keying; QoS: Quality of service; QPSK: Quadrature phase shift keying; RX: Receiver; SC-FDMA: Single carrier frequency division multiple access; SNR: Signal-to-noise ratio; TC-FSK: Turbo coded frequency shift keying; TC-OFDM: Turbo coded orthogonal frequency division multiplexing; TC-SC-FDMA: Turbo coded single carrier frequency division multiple access; TC: Turbo code; TX: Transmitter; UL: Uplink; ZC: Zadoff-Chu; ZF: Zero-forcing
\end{abstract}

\section{Acknowledgements}

The research leading to these results received funding from the European Commission H2020 program under grant agreement number 723247 (5G-Champion project). This work was also supported by the French Agence Nationale de la Recheche (ANR), under grant agreement ANR-16-CE25-0002 (project EPHYL).

\section{Authors' contributions}

YR and J-BD conceived of the presented idea. YR developed the theory and performed the computations. J-BD, LR, and VB verified the analytical methods. All authors discussed the results and contributed to the final manuscript. All authors read and approved the final manuscript.

\section{Competing interests}

The authors declare that they have no competing interests.

\section{Publisher's Note}

Springer Nature remains neutral with regard to jurisdictional claims in published maps and institutional affiliations.

\section{Author details}

${ }^{1}$ Univ. Grenoble Alpes, CNRS, Grenoble INP, GIPSA-lab, 38000 Grenoble, France. ${ }^{2}$ CEA, LETI, MINATEC Campus, 38054 Grenoble, France.

Received: 8 January 2018 Accepted: 13 June 2018

Published online: 03 July 2018

\section{References}

1. MR Palattella, M Dohler, A Grieco, G Rizzo, J Torsner, T Engel, L Ladid, Internet of Things in the $5 \mathrm{G}$ era: enablers, architecture, and business models. IEEE J. Sel. Areas Commun. 34(3), 510-527 (2016). https://doi.org/ 10.1109/JSAC.2016.2525418

2. ITU, Series Y: global information infrastructure, internet protocol aspects and next-generation networks next generation networks - frameworks and functional architecture models. https://www.itu.int/rec/T-REC-Y. 2060-201206-1

3. U Raza, P Kulkarni, M Sooriyabandara, Low Power Wide Area networks: an overview. IEEE Commun. Surv. Tutor. PP(99), 1-1 (2017). https://doi.org/ 10.1109/COMST.2017.2652320

4. HP Enterprise, Low Power Wide Area (LPWA) networks play an important role in connecting a range of devices. Business white paper (2016). http:// files.asset.microfocus.com/4aa6-5354/en/4aa6-5354.pdf

5. T Rebbeck, M Mackenzie, N Afonso, Low-powered wireless solutions have the potential to increase the M2M market by over 3 billion connections. Analysys Mason (2014). https://iotbusinessnews.com/download/whitepapers/ANALYSIS-MASON-LPWA-to-increase-M2M-market.pdf

6. Y Roth, J-B Doré, L Ros, V Berg, Turbo-FSK, a physical layer for low-power wide-area networks: analysis and optimization. Elsevier C. R. Phys. 18(2), 178-188 (2017). https://doi.org/10.1016/j.crhy.2016.11.005. Energy and radiosciences

7. FH Raab, P Asbeck, S Cripps, PB Kenington, ZB Popovic, N Pothecary, JF Sevic, NO Sokal, Power amplifiers and transmitters for if and microwave. IEEE Trans. Microw. Theory Tech. 50(3), 814-826 (2002). https://doi.org/10.1109/22.989965

8. H Ochiai, in Proceedings IEEE 56th Vehicular Technology Conference. Power efficiency comparison of OFDM and single-carrier signals, vol. 2, (2002), pp. 899-903. https://doi.org/10.1109/VETECF.2002.1040730

9. S Cui, AJ Goldsmith, A Bahai, Energy-constrained modulation optimization. IEEE Trans. Wirel. Commun. 4(5), 2349-2360 (2005). https:// doi.org/10.1109/TWC.2005.853882

10. SL Miller, RJ O'Dea, Peak power and bandwidth efficient linear modulation. IEEE Trans. Commun. 46(12), 1639-1648 (1998). https://doi. org/10.1109/26.737402

11. LTE Evolved Universal Terrestrial Radio Access (E-UTRA): physical channels and modulation. 3GPP TS 36.211, V13.2.0, Release 13 (2016). http://www. etsi.org/deliver/etsi_ts/136200_136299/136211/13.02.00_60/ts_ 136211v130200p.pdf

12. Whitepaper Narrowband Internet of Things. Rohde \& Schwarz (2016). https://cdn.rohde-schwarz.com/pws/dl_downloads/dl_application/ application_notes/1ma266/1MA266_0e_NB_loT.pdf

13. R Ratasuk, B Vejlgaard, N Mangalvedhe, A Ghosh, in 2016 IEEE Wireless Communications and Networking Conference Workshops (WCNCW). NB-loT System for M2M communication, (Doha, 2016), pp. 428-432. https://doi. org/10.1109/WCNCW.2016.7552737

14. YPE Wang, X Lin, A Adhikary, A Grovlen, Y Sui, Y Blankenship, J Bergman, HS Razaghi, A primer on 3GPP Narrowband Internet of Things. IEEE Commun. Mag. 55(3), 117-123 (2017). https://doi.org/10.1109/MCOM. 2017.1600510CM

15. C Berrou, A Glavieux, P Thitimajshima, in Communications, 1993. ICC'93 Geneva. Technical Program, Conference Record, IEEE International Conference on, vol.2. Near Shannon limit error-correcting coding and decoding: Turbo-codes. 1, (Geneva, 1993), pp. 1064-1070. https://doi.org/ 10.1109//CC.1993.397441

16. R Padovani, J Wolf, Coded Phase/Frequency Modulation. IEEE Trans. Commun. 34(5), 446-453 (1986). https://doi.org/10.1109/TCOM.1986. 1096564 
17. RA Khalona, GE Atkin, JL LoCicero, On the performance of a hybrid frequency and phase shift keying modulation technique. IEEE Trans. Commun. 41(5), 655-659 (1993). https://doi.org/10.1109/26.225476

18. A Latif, ND Gohar, Error rate performance of hybrid QAM-FSK in OFDM systems exhibiting low PAPR. Sci. China Ser F Inf. Sci. 52(10), 1875-1880 (2009). https://doi.org/10.1007/s11432-009-0165-y

19. SHong, M Sagong, C Lim, K Cheun, S Cho, in 2013 IEEE Globecom Workshops (GC Wkshps). FQAM : A modulation scheme for beyond $4 \mathrm{G}$ cellular wireless communication systems, (Atlanta, 2013), pp. 25-30. https://doi.org/10.1109/GLOCOMW.2013.6824956

20. Y Roth, The physical layer for low power wide area networks: a study of combined modulation and coding associated with an iterative receiver. PhD Thesis, Université Grenoble Alpes. (2017). https://hal.archivesouvertes.fr/tel-01568794

21. A Springer, W Gugler, M Huemer, L Reindl, CCW Ruppel, R Weigel, in IEEE/AFCEA EUROCOMM 2000. Information Systems for Enhanced Public Safety and Security (Cat. No.00EX405). Spread spectrum communications using chirp signals, (Munich, 2000), pp. 166-170. https://doi.org/10.1109/ EURCOM.2000.874794

22. B Reynders, S Pollin, in 2016 Symposium on Communications and Vehicular Technologies (SCVT). Chirp spread spectrum as a modulation technique for long range communication, (Mons, 2016), pp. 1-5. https://doi.org/10. 1109/SCVT.2016.7797659

23. LoRa Alliance. https://www.lora-alliance.org/. Accessed: 19 June 2018

24. OBA Seller, N Sornin, Low power long range transmitter. US 20140219329 A1, Aug 2014. https://patents.google.com/patent/US20140219329

25. L Vangelista, Frequency Shift Chirp Modulation: The LoRa Modulation. IEEE Signal Process. Lett. 24(12), 1818-1821 (2017)

26. D Chu, Polyphase codes with good periodic correlation properties (corresp.) IEEE Trans. Inf. Theory. 18(4), 531-532 (1972). https://doi.org/10. 1109/TIT.1972.1054840

27. MC Valenti, J Sun, The UMTS turbo code and an efficient decoder implementation suitable for software defined radios. Int J. Wireless Inf. Networks. 8, 203-216 (2001)

28. Z Wang, GB Giannakis, Wireless multicarrier communications. IEEE Signal Proc. Mag. 17(3), 29-48 (2000). https://doi.org/10.1109/79.841722

29. Y Roth, JB Doré, L Ros, V Berg, in 2015 IEEE 16th International Workshop on Signal Processing Advances in Wireless Communications (SPAWC). TurboFSK: a new uplink scheme for Low Power Wide Area networks, (Stockholm, 2015), pp. 81-85. https://doi.org/10.1109/SPAWC.2015.7227004

30. CE Shannon, A mathematical theory of communication. Bell Syst. Tech. J. 27(3), 379-423 (1948). https://doi.org/10.1002/j.1538-7305.1948. tb01338.x

31. JG Proakis, Digital Communications 3rd Edition. Communications and signal processing. (McGraw-Hill, New York, 1995)

32. Y Roth, J-B Doré, L Ros, V Berg, in 20169 th International Symposium on Turbo Codes and Iterative Information Processing (ISTC). EXIT chart optimization of Turbo-FSK: Application to Low Power Wide Area networks, (Brest, 2016), pp. 46-50. https://doi.org/10.1109/ISTC.2016.7593074

33. (WC Jakes, DC Cox, eds.), Microwave Mobile Communications. (Wiley-IEEE Press, Hoboken, 1994)

34. Evolved Universal Terrestrial Radio Access (E-UTRA): Base Station (BS) Radio Transmission and Reception. 3GPP TS 36.104, V14.3.0, Release 14 (2017). http://www.etsi.org/deliver/etsi_ts/136100_136199/136104/14. 03.00_60/ts_136104v140300p.pdf

35. P Bento, J Nunes, M Gomes, R Dinis, V Silva, in 2014 IEEE 80th Vehicular Technology Conference (VTC2014-Fall). Measuring the magnitude of envelope fluctuations: should we use the papr? (Vancouver, 2014), pp. 1-5. https://doi.org/10.1109/NTCFall.2014.6966053

36. V Mannoni, V Berg, F Dehmas, D Noguet, A flexible physical layer for LPWA cognitive radio oriented wireless networks. Crown Com 2017, vol. 228. (Springer, Cham, 2018). https://doi.org/10.1007/978-3-319-76207-4_27

\section{Submit your manuscript to a SpringerOpen ${ }^{\circ}$ journal and benefit from:}

- Convenient online submission

- Rigorous peer review

- Open access: articles freely available online

- High visibility within the field

- Retaining the copyright to your article

Submit your next manuscript at $>$ springeropen.com 